\title{
Higher order dynamic mode decomposition to identify and extrapolate flow patterns
}

\author{
Soledad Le Clainche ${ }^{a)}$ and José M. Vega \\ E.T.S.I. Aeronáutica y del Espacio, Universidad Politécnica de Madrid, 28040 Madrid, Spain
}

\begin{abstract}
This article shows the capability of using a higher order dynamic mode decomposition (HODMD) algorithm both to identify flow patterns and to extrapolate a transient solution to the attractor region. Numerical simulations are carried out for the three-dimensional flow around a circular cylinder, and both standard dynamic mode decomposition (DMD) and higher order DMD are applied to the nonconverged solution. The good performance of HODMD is proved, showing that this method guesses the converged flow patterns from numerical simulations in the transitional region. The solution obtained can be extrapolated to the attractor region. This fact sheds light on the capability of finding real flow patterns in complex flows and, simultaneously, reducing the computational cost of the numerical simulations or the required quantity of data collected in experiments. Published by AIP Publishing. [http://dx.doi.org/10.1063/1.4997206]
\end{abstract}

\section{INTRODUCTION}

Dynamic mode decomposition (DMD) is a technique introduced by Schmid ${ }^{23}$ that uses the Koopman linear operator $^{11}$ to calculate Fourier-like expansions for non-linear dynamics. The strength of this technique mainly lies in its capability to reconstruct the original data set analyzed by means of identifying its flow dynamics, which also allows to study its main spatio-temporal flow patterns and physical insight. Therefore, with such aim, DMD is applied to a set of $K$ time equispaced spatio-temporal snapshots relying in the following Koopman assumption, which relates each snapshot $\boldsymbol{v}_{k+1}$ (calculated at the time instant $t_{k+1}$ ) with the previous snapshot $\boldsymbol{v}_{k}$ (calculated at time instant $t_{k}$ ) by means of the Koopman operator $\boldsymbol{R}$, as

$$
\boldsymbol{v}_{k+1}=\boldsymbol{R} \boldsymbol{v}_{k} \quad \text { for } k=1, \ldots, K-1 .
$$

Based on this idea, after some calculations (detailed below), it is possible to reconstruct the set of $K$ timeequispaced data in the sampled time interval $t_{1} \leq t \leq t_{1}+T$ using the following spatio-temporal expansion of modes:

$$
\begin{gathered}
\mathrm{v}(\boldsymbol{x}, t) \diamond \mathrm{v}^{\mathrm{DMD}}(\boldsymbol{x}, t) \equiv{ }_{m=1}^{I^{\prime} 1} a_{m} u_{m}(x) \mathrm{e}^{\left(\delta_{m}+\mathrm{i} \omega_{m}\right) t} \\
\text { for } t_{1} \leq t \leq t_{1}+T,
\end{gathered}
$$

where the spatial fields $u_{m}(\boldsymbol{x})$ are known as DMD modes, and $a_{m}, \delta_{m}$, and $\omega_{m}$ are their corresponding associated amplitudes, growth rates, and frequencies. If the time interval $\left(t_{1}, t_{1}+T\right)$ in which the data are first calculated, and next reconstructed, is substituted by any interval more advanced in time $\left(t_{r}, t_{r}+T\right)$, with $t_{r} \gg t_{1}$, the same expansion can be used to extrapolate the original data to the attractor region, where the dynamics are permanent $\delta_{m}=0$ (saturated flow).

\footnotetext{
a) Author to whom correspondence should be addressed: soledad.leclainche@ upm.es
}

The spatial and temporal complexity and dimension are also relevant parameters in the previous expansion (2). On the one hand, the number of expansion terms, $M$, can be referred to as the spectral or temporal complexity, while the temporal dimension, denoted by $K$, is dependent on the number of data collected in time; thus the length of the time interval $t_{1} \leq t$ $\leq t_{1}+T$, where $T=K \cdot \Delta t$ (time-equispaced data). On the other hand, the length of the spatial vector $\boldsymbol{x}$ determines the spatial dimension $J$, while the spatial complexity is determined by the dimension of the subspace generated by the DMD modes, as

$$
N=\operatorname{dim}\left(\operatorname{span}\left\{\boldsymbol{u}_{1}, \ldots, \boldsymbol{u}_{M}\right\}\right) \leq \min \{M, J\} .
$$

If the spatial and temporal complexities are equal, $N$ $=M$, conditions (1) and (2) are exact, meaning that the performance of the standard DMD is optimal. The good performance of DMD has been evidenced several times in the literature either to study flow dynamics in numerical and experimental data ${ }^{12,13,24}$ or to compute the global linear modes in linear flows. $5,7,20,22$

However, when the spatial complexity is smaller than the spectral complexity, $N<M$, the previous assumption is not valid. Instead, it is necessary to introduce a higher order dynamic mode decomposition (HODMD) approximation or DMD-d algorithm. This higher order technique has been recently introduced by Le Clainche and Vega, ${ }^{16}$ as an extension of the classical DMD that is capable of providing highly accurate results in cases in which the performance of the classical DMD is deteriorated or even fails. HODMD mixes the ideas behind classical DMD with Takens' delay embedding theorem, ${ }^{25}$ leading to a higher order Koopman assumption that uses time-lagged snapshots, as

$$
\begin{aligned}
\boldsymbol{v}_{k+d}= & \boldsymbol{R}_{1} \boldsymbol{v}_{k}+\boldsymbol{R}_{2} \boldsymbol{v}_{k+1}+\cdots+\boldsymbol{R}_{d} \boldsymbol{v}_{k+d-1} \\
& \text { for } k=1, \ldots, K-d,
\end{aligned}
$$

and is used to calculate the DMD expansion proposed in Eq. (2). Let us note that $d \geq 1$ is tunable and, when $d=1$ 
(i.e., applying DMD-1), this assumption exactly matches the standard Koopman assumption presented in Eq. (1). In this way, it is possible to relate DMD-d algorithm or HODMD with classical $\mathrm{DMD},{ }^{23}$ which in this article is defined as DMD-1.

While the temporal complexity $M$ is only dependent on the (spectral) complexity of the real data (flow dynamics), both the spatial and temporal dimensions, $J$ and $K$, are dependent of the type of data analyzed. For example, if DMD analysis is performed to study the results of a three-dimensional numerical simulation, the spatial dimension $J$ is determined by the total number of grid points defining the computational domain, as

$J=n_{x} \times n_{y} \times n_{z}$, while the number of $K$ equispaced temporal data collected is a choice of the user, more related to the time step of the numerical simulation and the computer's storage memory. As expected, the spatial dimension is decreased in the

case of two-dimensional numerical simulations $\left(J=n_{x} \times n_{y}\right)$ and may be drastically decreased in the case of experiments. For example, particle image velocimetry (PIV) experimental measurements are usually performed on a small plane in which

the spatial dimension is $J=n_{x} \times n_{y}$ (smaller than in a numerical simulation). Similarly, in hot wire (HW) measurements, the spatial dimension $J$ is equal to the number of HW probes used in the experiment. Also, the temporal dimension $K$ may be determined by the type of experiment performed, since in complex experiments, the number of data collected is restricted by some external factors (i.e., wall confined flow with thermal effects ${ }^{15}$ ).

A different issue is the calculation of the spatial complexity $N$, in which, naturally, the spatial dimension $J$ of the original data and their temporal complexity $M$ play a highly essen-

tial role $[N \leq \min \{M, J\}$, see Eq. (3)]. However, some other external factors, such as noise or transient decaying dynamics, may also affect this variable. Thus, it is necessary to analyze carefully the parameter $N$ and the sensitive factors that may contribute to alter it, leading to a spatial complexity that is smaller than the spectral complexity. In such cases, HODMD must be used instead of the classical algorithm. It is possible to mention three natural cases with larger spectral complexity than spatial complexity, $N<M$ :

(i) When the spectral complexity $M$ is very large but the number $J$ of collected data is limited, either due to (a) the number of involved differential equations (in numerical simulations) or due to (b) the number of measurements points coming from an experiment: Since the spatial dimension $J$ is small, $N$ is small. Case (a) is commonly found in non-linear dynamical systems and becomes determined by the definition of the problem. For example, in a periodic solution of the Lorenz equation or in a quasi-periodic solution of the GinzburgLandau equation, one finds that $N<M$ (Le Clainche and Vega ${ }^{16}$ ). Case (b) is also usually found in several types of experiments used for industrial purposes such as the flight tes $\mathrm{t}^{18}$ or in the analysis of magnetic resonances.

(ii) When the data are noisy: This is a case usually found in experimental data. Even in simple flow dynamic cases in which ideally $N=M$, the noise found in the spatial structure defining the data needs to be calculated first in order to be cleaned next. So, some of the spatial modes are used to calculate this noise, leading to the modification of the real calculations of spectral and spatial complexities and, consequently, producing that the effective value of $N$ be smaller than $M$ (Le Clainche et $a l .{ }^{17}$.

(iii) When the data analyzed come from a transitory stage: This case may be represented by the Stuart-Landau equation ${ }^{1}$ and is a case usually found in non-converged numerical simulations, or in the transitory stage of an experiment. Even though the converged dynamics may be represented by an equal value of the spatial and temporal complexities $(N=M)$, when DMD analysis is performed in a transitory region, some of the dynamics describing the flow are decaying $\left(\delta_{m}<0\right)$. Thus, as in the previous item, some of the spatial modes are used to calculate these decaying modes, modifying the actual values of the spectral and spatial complexities and, consequently, leading to $N<M$.

Cases (i) and (ii) are related to interpolation (reconstruction of the original data) and are already explained in detail in the literature. ${ }^{16,17}$ Case (iii), which is more related to pattern identification and extrapolation, is still an open topic that will be addressed in this article. If it were possible to calculate the main dynamics describing the flow in the transient region and to construct the DMD expansion proposed in Eq. (2), it would be (in turn) possible to extrapolate the solution to the attractor region by only retaining the non-decaying DMD modes.

In this article, the transient regime of the threedimensional flow around a circular cylinder is analyzed using HODMD, with the aim to show the good performance of this technique both to calculate the real flow patterns in nonconverged solutions and its high potential to be used as a reduced order model for extrapolation. The latter is useful either to reduce the computational cost in numerical simulations or to reduce the time and the number of data collected in experimental measurements, which sometimes, due to their high complexity, are subject to external, out of control restrictions. ${ }^{15}$

The article is organized as follows. The above mentioned extension of standard DMD, called HODMD, is briefly described in Sec. II. The cylinder wake test problem will be considered in Sec. III, where the numerical method to simulate the flow around the cylinder will also be described. The main results of the paper are given in Sec. IV, where both classical DMD and higher order DMD will be applied to guess the dynamics on the attractor from results computed in the transient region, comparing both the relevant involved frequencies and the flow patterns. The paper ends with some concluding remarks, in Sec. V.

\section{HIGHER ORDER DYNAMIC MODE DECOMPOSITION ALGORITHM}

The algorithm presented in this section is explained more in detail in Ref. 16. As anticipated, we consider a data set of $K$ equispaced snapshots, collected in a snapshot matrix $V_{1}^{K}$ (whose columns are the snapshots, from the first to the $K$ th 
snapshot) in the following way:

$$
\boldsymbol{V}^{K}=\left[\boldsymbol{v}_{1}, \ldots, \boldsymbol{v}_{k}\right]
$$

where $\boldsymbol{v}_{k}$ is the vector representing the spatio-temporal data (snapshot) collected at time instant $t_{k}$. The HODMD algorithm is applied to this snapshot matrix to study the main flow dynamics and to represent the spatio-temporal snapshot as the following DMD expansion [same as in Eq. (2)]:

$$
\begin{aligned}
& \mathrm{V}(\boldsymbol{x}, t) \diamond \mathrm{v}^{\mathrm{DMD}}(\boldsymbol{x}, t) \equiv{ }_{m=1}^{I^{M} 1} a_{m} u_{m}(\boldsymbol{x}) \mathrm{e}^{\left(\delta_{m}+\mathrm{i} \omega_{m}\right) t} \\
& \quad \text { for } t_{1} \leq t \leq t_{1}+T .
\end{aligned}
$$

HODMD, using the DMD- $d$ algorithm, considers two main steps:

1. Singular value decomposition (SVD) is applied to the snapshot matrix $V_{1}^{K}$, leading to a representation of the spatio-temporal data as an expansion of spatial and temporal modes, $\boldsymbol{U}$ and $\boldsymbol{T}$, respectively, and singular values $\Sigma$, written in a matrix form as

$$
\boldsymbol{V}_{1}^{K} \diamond \boldsymbol{U} \Sigma \boldsymbol{T}^{\top}, \equiv \boldsymbol{U} \hat{\boldsymbol{V}}_{1}^{K} \text {, with } \hat{\boldsymbol{V}}_{1}^{K}=\Sigma \boldsymbol{T}^{\top}\left(\equiv \boldsymbol{U}^{\top} \boldsymbol{V}_{1}^{K}\right)
$$

A dimension reduction of the spatial terms is carried out in this step, retaining $N$ (=spatial complexity) spatial modes. The value of $N$ is selected to ensure that the SVD approximation satisfies a certain root mean square (RMS) error (selected by the user), as

$$
\operatorname{EE}(N) \equiv \frac{\sigma_{N^{+1}}^{2}+\cdots+\sigma_{R}^{2}}{\sigma_{1}^{2}+\cdots+\sigma_{R}^{2}} \leq \varepsilon_{1},
$$

where $R \leq \min \{J, K\}$ is the rank of the snapshot matrix, $\sigma_{i}$ are the singular values (contained in matrix $\Sigma$ ) sorted in decreasing order, and $\varepsilon_{1}$ is a parameter tuneable by the user to control the error of the approximation. In the case of noisy data or unconverged solutions, this parameter could be compared to the uncertainty contained in the data. ${ }_{\text {Equation (7) implies that the size of the snapshots }}^{17}$

$\boldsymbol{v}_{k}$ is reduced to $\hat{\boldsymbol{v}}_{k}$ in the following way:

$$
\boldsymbol{v}_{k}=\boldsymbol{U} \hat{\boldsymbol{v}}_{k} .
$$

When the data collected are too noisy, as in the case of an experiment, a higher order singular value decomposition (HOSVD) ${ }^{4,10,26}$ is used instead of SVD at this step. ${ }^{17}$ The HOSVD algorithm performs a SVD in each one of the spatial directions. Thus, in this way, it is possible to better clean the data from noise or to remove non-permanent modes $\left(\delta_{m}<0\right)$, which somehow could be considered as noise (snapshots reduction).

2. The DMD- $d$ algorithm (with $d \geq 1$ ) is applied to the temporal modes $\hat{\boldsymbol{V}}_{1}^{K}$ (the reduced snapshot matrix) obtained in the previous step. Therefore, the higher order Koopman assumption presented in Eq. (4) is used in the reduced snapshots $\hat{\boldsymbol{v}}_{k}$ (contained in a reduced linear manifold) as

$$
\begin{aligned}
\hat{\boldsymbol{v}}_{k+d} \diamond \hat{\boldsymbol{R}}_{1} \hat{\boldsymbol{v}}_{k}+\hat{\boldsymbol{R}}_{2} \hat{\boldsymbol{v}}_{k+1}+\cdots+\hat{\boldsymbol{R}}_{d} \hat{\boldsymbol{v}}_{k+d-1} \\
\text { for } k=1, \cdots, K-d .
\end{aligned}
$$

The remaining of this step, in turn, considers two cases, depending on the value of $d$ :

(a) If $d=1$ : DMD-1 algorithm.

The standard higher order Koopman assumption (10) is used in the dimension reduced snapshot as

$$
\hat{\boldsymbol{v}}_{k+1} \diamond \hat{\boldsymbol{R}}_{1} \hat{\boldsymbol{v}}_{k} \text {, for } k=1, \ldots, K-1 .
$$

In the matrix form,

$$
\hat{\boldsymbol{V}}_{2} \hat{\boldsymbol{R}}^{\mathrm{R}} \hat{\boldsymbol{V}}_{1}^{K-1}, \text { with } \hat{\boldsymbol{R}}=\boldsymbol{U}^{\top} \boldsymbol{R} \boldsymbol{U} \text {. }
$$

Let us note that this equation is similar to the classical Koopman assumption presented in Eq. (3), but applied to the dimension reduced snapshot. Thus, the DMD-1 algorithm is similar to classical DMD. ${ }^{23}$

Then, SVD is applied to the snapshot matrix $\hat{\boldsymbol{V}}_{1}^{K-1}$ to perform its pseudo-inverse, and the linear operator $\hat{\boldsymbol{R}}$ is calculated. This operator contains the dynamics of the system. So, its eigenvectors and eigenvalues yield the modes $u_{m}(\boldsymbol{x})$, growth rates $\delta_{m}$, and frequencies $\omega_{m}$ appearing in the DMD expansion (6). The mode amplitudes $a_{m}$ are calculated upon least

squares fitting. A second tolerance $\varepsilon$ is set in order to retain the $M$ most relevant DMD modes, exhibiting the largest amplitudes $a_{m}$. This parameter will determine the spectral complexity of the DMD expansion (2), calculated for a specific accuracy $\varepsilon$ (tunable by the user).

(b) If $d>1$ : DMD-d algorithm.

The higher order Koopman assumption is used as in Eq. (10). This equation is written as the following modified Koopman equation:

$$
\tilde{\boldsymbol{v}}_{k+1} \diamond \tilde{\boldsymbol{R}} \tilde{\boldsymbol{v}}_{k},
$$

where the modified snapshots $\tilde{\boldsymbol{v}}_{k}$ and the modified Koopman matrix $\tilde{\boldsymbol{R}}$ are

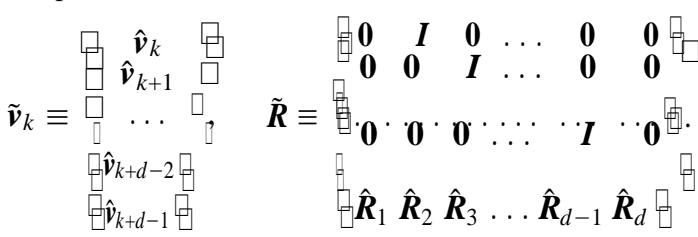

The modified Koopman operator $\boldsymbol{R}$ is calculated as in the previous case, and its eigenvectors and eigenvalues yield the modes $u_{m}(\boldsymbol{x})$, growth rates $\delta_{m}$, and frequencies $\omega_{m}$ of the DMD expansion (6). Again, the mode amplitudes $a_{m}$ are calculated upon least squares fitting, and a second tolerance $\varepsilon$ is set in order to retain the $M$ most relevant DMD modes, exhibiting the largest amplitudes $a_{m}$. This parameter will determine the spectral complexity of the DMD expansion (2), calculated for a specific accuracy $\varepsilon$ (tunable by the user).

The benefit of this method lies in the resolution of the eigenvalue problem for the modified Koopman matrix $\tilde{\boldsymbol{R}}$, which enforces the dynamic contained in the delayed snapshot matrix to evoke the same solution. So, either the noise or non-permanent devices are naturally removed from the solution. 


\section{THE THREE-DIMENSIONAL CYLINDER WAKE}

Let us now consider a classical fluid mechanics case, namely, the incompressible three-dimensional cylinder wake. ${ }^{28}$ For large aspect ratio cylinders, the spatio-temporal structure of the wake only depends on the Reynolds number, defined as $\operatorname{Re}=U_{\infty} D / \mathrm{v}$, where $U_{\infty}$ is the incoming free stream velocity, $D$ is the cylinder diameter, and $\mathrm{v}$ is the kinematic viscosity. At small Re, the wake is two-dimensional and steady, but at Re 46, it exhibits a primary Hopf bifurcation, ${ }^{,, 21}$ which still produces a two-dimensional but unsteady (in fact, periodic) von Karman vortex street flow. This periodic flow remains orbitally stable up to Re 190, where it suffers a secondary bifurcation (Floquet multiplier $=1$ ) to three-dimensional periodic oblique waves, as repeatedly found experimentally in large aspect ratio containers. ${ }^{8,27}$ This threshold was (more precisely) calculated numerically via Floquet analysis by Barkley and Henderson, ${ }^{2}$ as (critical Reynolds number) $\operatorname{Re}^{c}=188.5 \pm 1.0$, by imposing periodicity in the

span direction, with wavenumber $\beta$ (associated spatial period $L_{z}=2 \pi / \beta$ ), whose critical value at $\operatorname{Re}=\operatorname{Re}^{c}$ was found to

be $L_{z}^{c}=3.96 \pm 0.02$. For $\operatorname{Re} \geq \operatorname{Re}^{c}$, Barkley and Henderson estimated the frequency of the spanwise pattern as that associated with the most unstable mode, known as mode $A$, which has been studied in more detail by Blackburn et al. ${ }^{3}$ In particular, for $\mathrm{Re}=220$ (the case that will be considered below), the experimental spatial period (estimated by Barkley and Henderson ${ }^{2}$ from the experimental visualizations of Williamson ${ }^{27}$ ) and temporal frequency (measured by Williamson ${ }^{27}$ ),

$$
L_{z}=4.01, \quad f_{\exp } \diamond .185 \quad\left(\text { or } \omega_{\exp }=2 \pi / f_{\exp } \diamond 1.1624\right),
$$

can be taken as reference. For this value of $L_{z}$, the Floquet analysis in Barkley and Henderson ${ }^{2}$ yielded the frequency

$f \diamond 0.20 \mathrm{~Hz}$ (or $\omega \diamond 1.2566$ ), which compares reasonably well with its experimental counterpart [see Eq. (15)]. With this background in mind, we shall use the HODMD method to analyze the cylinder at $\mathrm{Re}=220$ using numerically generated data via a computational fluid dynamics (CFD) tool.

The numerical code used is Nek5000, ${ }^{19}$ an open source CFD code that uses spectral elements as spatial discretization. The three-dimensional incompressible Navier-Stokes equations has been solved, in a Cartesian reference frame aligned with the streamwise, vertical, and spanwise directions, $x, y$, and $z$, respectively. The dimensions of the computational domain coincide with those used in Barkley and Henderson, ${ }^{2}$ namely, $\mathrm{x} \in[-15,25], \mathrm{y} \in[-20,20]$, and $\mathrm{z} \in[0,4]$, respectively; the axis of the cylinder (whose nondimensional diameter is $D$ $=1$ ) is the straight line $x=y=0$. The mesh contains 1132 elements and was generated using the open source code Gmsh. ${ }^{6}$ Finally, each element was discretized using Gauss-LobattoLegendre points of order $p=5$. A grid convergence study has been performed first, in a two dimensional mesh, in order to set the polynomial order to 5 . Then, FFT has been applied to several points in the computational domain, finding in all cases that the dominant frequency was $f_{2 d} \diamond 0.20\left(\omega_{2 d}\right.$ $=1.2566)$, similar to the frequency $f$ calculated by Barkley and Henderson. ${ }^{2}$ In order to reduce the computational cost, the three-dimensional numerical simulation was initialized with this two-dimensional converged solution. The boundary conditions (b.c.) set in the cylinder surface was no-slip. In the inlet surface, it was enforced uniform flow $\left(u_{x}=U_{\infty}=1, u_{y}=0\right)$, while at the top and bottom parts of the domain and the outlet surface, the b.c. used were standard outflow conditions ( $p$ $\left.=0, \partial_{x} u_{x}=0, \partial_{x} u_{y}=0\right) \cdot{ }^{14} \mathrm{~A}$ second test case was carried out setting uniform b.c. (as in the inlet surface) in the top and bottom surfaces, as in Barkley and Henderson ${ }^{2}$ and the results obtained were similar. This fact proves that the boundaries of the computational domain are sufficiently far from the cylinder studied, minimizing their effects over the solution presented. Finally, in the three-dimensional expansion in the spanwise direction, the b.c. were set to periodic (Fourier expansion), with a period $L_{z}=4$, which is consistent with the expected period (15) for this value of the Reynolds number ${ }_{3}$ The time step in the numerical simulations was set to $5 \cdot 10$, in order to maintain the Courant-Friedrichs-Lewy condition (CFL) in a value smaller than 1 . The numerical code was evolved in time during 1325 time units, saving snapshots (each containing the 1132 end-points of the 1132 elements) every $\Delta t=0.5$ time units, meaning that we have stored a total number of 2650 snapshots. However, discarding the transient $0 \leq t \leq 575$, only the last 1500 snapshots (750 time units) will be used below. Because the considered value of the Reynolds number is not too far from its threshold value for the transition to three-dimensional dynamics, the considered snapshots are not expected to be fully converged to the final periodic attractor, which is found after evolving the code in time during 2900 time units. The dominant frequency calculated in the attractor was $\omega_{\text {num }}=1.1711$, comparable to the spanwise velocity obtained in the experiment (15). Figure 1 shows the time evolution of the residuals in the three-dimensional numerical simulations in a representative point of the computational domain (cylinder wake). As seen, the fact that the Navier-Stokes equations are initialized with the converged solution of the two dimensional case is reflected in this velocity evolution, in which it is possible to identify the growth of the spanwise mode.

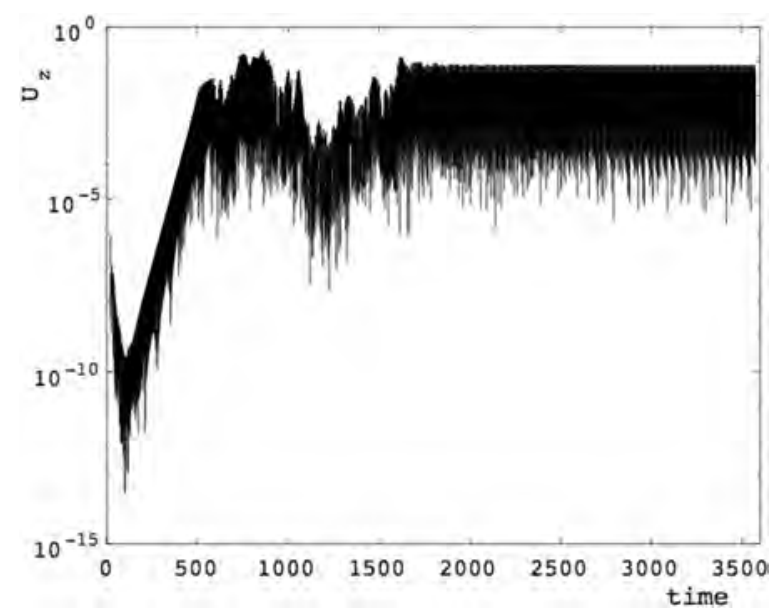

FIG. 1. Evolution of spanwise velocity in the three-dimensional numerical simulations, taken in the cylinder wake at $(x, y, z)=(2,0,0)$ [the cylinder is located at $(0,0, z)]$. 


\section{PATTERN FORMATION AND EXTRAPOLATION TO THE ATTRACTOR REGION}

We perform various sets of HODMD computations, restricting the data to the plane $y=0$ and using the three velocity components [to somewhat mimic stereo particle image velocimetry (PIV) data] and considering both the near-field and far-field in the streamwise direction, namely,

$$
\begin{aligned}
& y=0 \text {-near-field : } \quad 0.5 \leq x \leq 4, y=0,0 \leq z \leq 4, \\
& y=0 \text {-far-field : } \quad 4 \leq x \leq 25, y=0,0 \leq z \leq 4,
\end{aligned}
$$

and two sets of tolerances, namely,

$$
\begin{array}{r}
\text { rough: } \varepsilon_{1}=10^{-2}, \varepsilon=2 \cdot 10^{-2} \\
\text { more precise: } \varepsilon_{1}=10^{-4}, \varepsilon=3 \cdot 10^{-3} .
\end{array}
$$

Additional computations have been performed using (a) only the in-plane velocities in the $y=0$-near field data and (b) the whole three-dimensional data in the near field. As expected, those results (omitted here for the sake of brevity) are slightly worse in case (a) and slightly better in case (b), compared with the results that will be presented in this section.

In all applications below, the extrapolation properties of both DMD-1 and DMD- $d$ will be evidenced. The analyses have been performed in two different sets of data, using the rough and more precise tolerances. In the first case, we collect a set of 500 snapshots, starting at time 575, sufficiently far from the attractor (time 2900) to prove the benefits of extrapolating instead of integrating Navier-Stokes equations, in terms of reduction of computational time. In the second case, we collect a set of 1000 snapshots, starting at time 875 in order to show that, when the distance to the attractor is reduced, DMD- $d$ is capable of giving a better prediction of the flow patterns of the attractor.

Let us start with the first application, in which both DMD1 and DMD-250 are applied to the set of 500 snapshots. The value $d=250$ has been selected after some calibration (looking for consistency and robustness in the results presented ${ }^{17}$ ). As in the remaining applications of the HODMD method described above, this value of $d$ can be decreased by 50 without significant changes in the performance of the method. In this first application of the method, the rough tolerances are used in the near field. The plots of the damping rates and mode amplitudes vs. the retained frequencies are given in Fig. 2. The following can be observed in these plots:

- With these tolerances, DMD-250 identifies two kinds of modes: transient $(\delta<0)$ and permanent $(\delta \diamond 0)$ modes. Since the $\omega=0$ (mean flow) and ten pairs of complex conjugate modes (plotted with blue circles) exhibit a damping rate of $\sim 2 \cdot 10^{3}$ and thus are useful for extrapolation to estimate the periodic attractor, these modes will be called the permanent modes below. There is a small gap that separates permanent modes from the remaining 19 pairs of modes to be called transient modes below (candidates to be eliminated in extrapolation), plotted with blue crosses.

Besides, the permanent modes include the experimentally measured fundamental frequency with a relative error $\sim 10^{-3}$, and their second, third, and fourth harmonics. In addition, somehow unexpectedly, DMD-250 also identifies a 1/3-subharmonic of the experimental frequency. Moreover, the exact commensurability relations for these harmonics and subharmonics hold within a maximum relative error of $\sim 7 \cdot 10^{3}$.

- The performance of DMD-1 could be seen as qualitatively similar, since it produces a similar gap between permanent and transient modes. However, DMD-1 only identifies the mean flow and four pairs of complex conjugate modes (of 240 modes calculated in total) including the experimental frequency (with a relative error of $\sim 5 \cdot 10^{3}$ ), its harmonic, and the $1 / 3$-subharmonic mode previously presented.

The results above raise the question on whether the 1/3subharmonic identified above by DMD-250 is the relevant fundamental frequency (though with a much smaller mode amplitude than the experimentally detected frequency), and thus, strictly speaking, the period of the attractor is three times larger than assumed. This issue is first analyzed considering the precise tolerances in Eq. (18), which gives the results plotted in Fig. 3 (a second analysis will be done below by using data from the fully converged attractor). As can be seen in the following:

- Though the gap between permanent and transient modes is similar than in Fig. 2, the amount (namely, 15) of permanent modes identified by DMD-250 is larger, their growth rate is smaller $\left(\sim 10^{3}\right)$, and the amount of transient modes is larger. DMD-250 identifies as permanent modes the mean flow and 7 pairs of complex conjugate modes, which include the "fundamental frequency" (with a relative error of $\sim 1.9 \cdot 10^{3}$ ) plus two harmonics of the fundamental
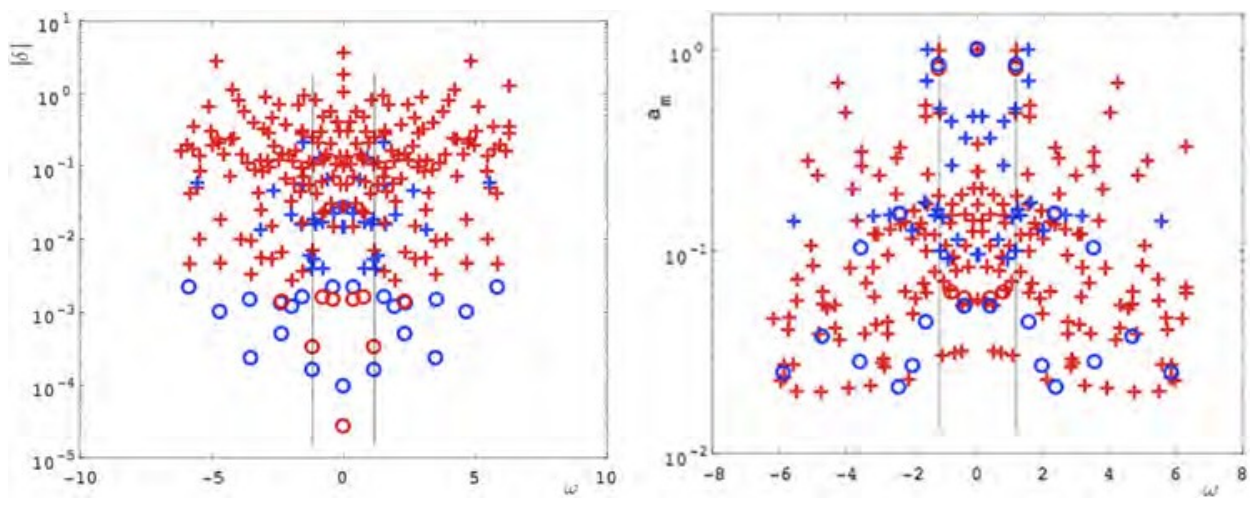

FIG. 2. The $y=0$-near field defined in Eq. (16) with the rough tolerances in Eq. (18), considering the three velocity components: damping rates (left) and mode amplitudes (right) vs. the retained frequencies obtained via DMD250 (blue symbols) and DMD-1 (red symbols) applied in a set of 500 snapshots starting at time 575 . Those modes exhibiting the smaller damping rates $(\delta \diamond 0)$ are plotted with circles, while the remaining modes are plotted with crosses. The experimental frequencies $\pm \omega_{\text {exp }}$ [see Eq. (15)] are indicated with vertical lines. 

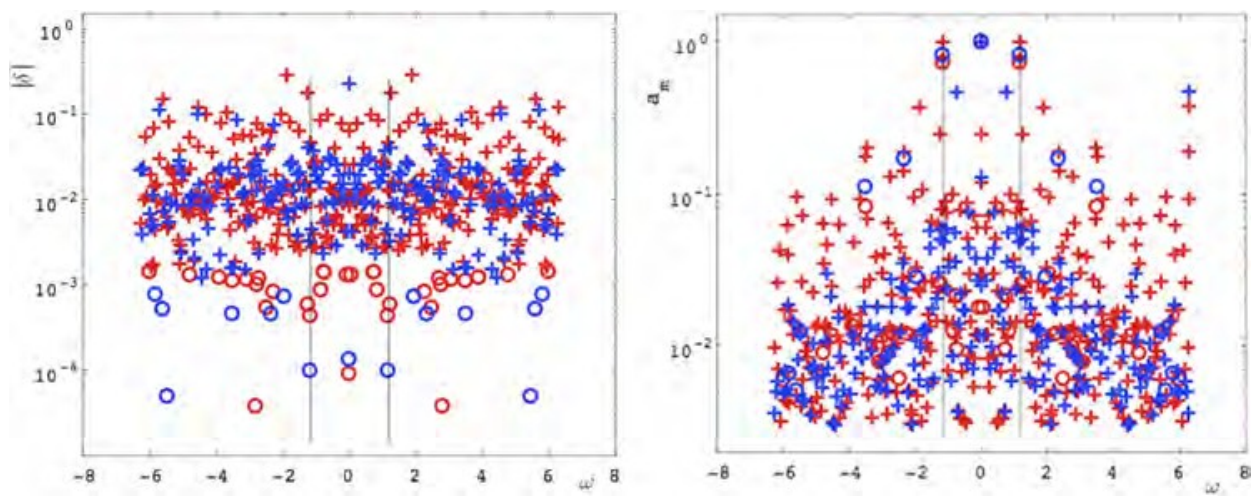

FIG. 3. Counterpart of Fig. 2, but considering the more precise tolerances defined in Eq. (18).

frequency and some harmonics of the 1/3-subharmonic (excluding this mode), with the exact commensurability relations holding within a maximum relative error of $\sim 6 \cdot 10^{3}$.

- Concerning DMD-1, it is possible to identify two gaps related to the permanent-transient mode division. The first gap includes the mean flow and a pair of conjugate modes that correspond to the second harmonic of the experimental frequency. The second gap also includes 14 pairs of conjugate modes corresponding to both sub-harmonics and harmonics of such experimental frequency. The fact that the experimental frequency is not identified in the first gap makes it necessary to consider the second gap as the identifier of the permanent modes, which is somewhat confusing in practice since, in contrast to HODMD, it requires knowledge of the right involved modes.

Let us now compare the extrapolation results computed above with their counterparts computed in the attractor itself. Figure 4 shows the attractor frequencies and amplitudes calculated at time 2900. As expected, similar results are obtained in a three-dimensional domain and in the near and far field planes defined in Eqs. (16) and (17), using DMD-1, DMD-d (various $\mathrm{d}$ ), several tolerances, and different numbers of snapshots. As seen, the $1 / 3$-subharmonic mode is also found in this region, but with a small amplitude. Table I shows the

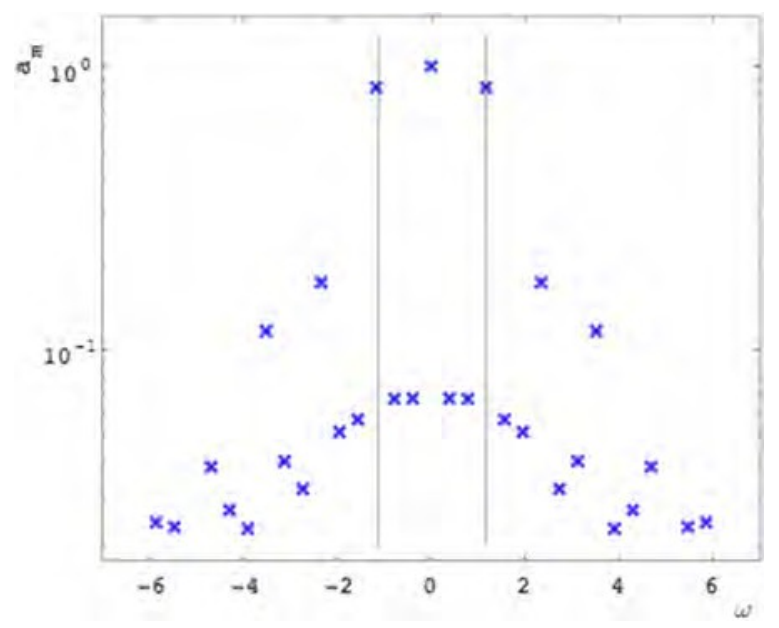

FIG. 4. Attractor amplitudes vs. retained frequencies (time 2900). frequencies presented in Fig. 2 (extrapolation), Fig. 3 (extrapolation), and Fig. 4 (attractor). The results in this table show that, on the one hand, DMD-250 captures a larger number of relevant frequencies than DMD-1, especially in the case calculated with the rough tolerances in Eq. (18). However, the method also doubles a couple of frequencies. On the other hand, the presence of the 1/3-subharmonic mode is found in all cases, which fully confirm that this unexpected subharmonic is not an artifact. Regarding these results, it is possible to say that the performance of DMD-250 is slightly better than the performance of classical DMD, making HODMD a suitable choice to be used for extrapolation, since it calculates a larger number of frequencies, with higher accuracy. Additionally, the issue of doubling certain frequencies may not affect the final results if the purpose of applying this method is either the reconstruction of the original data (free from transient modes, data cleaned from noise) or the extrapolation to the attractor, since these modes are multiplied by their amplitudes, which makes up for this division. Yet, if the method is only used for studying structural patterns, this fact may lead to some misunderstandings.

With the aim of showing the robustness of HODMD and the calibration process carried out, the same calculations

TABLE I. Summary of the permanent frequencies $\omega$ identified in Figs. 2 and 3 and in the attractor (Fig. 4). Reference frequency (experiment): $\omega_{\text {exp }}$ $=1.1624$.

\begin{tabular}{|c|c|c|c|c|}
\hline \multicolumn{2}{|c|}{ Rough tolerance (18) } & \multicolumn{2}{|c|}{ More precise tolerance (18) } & \multirow{2}{*}{$\frac{\text { Attractor }}{\ldots}$} \\
\hline DMD-1 & DMD-d & DMD-1 & DMD-d & \\
\hline 0.3852 & 0.3869 & 0.3852 & & 0.3904 \\
\hline 0.7819 & & 0.7819 & & 0.7807 \\
\hline \multirow[t]{3}{*}{1.1648} & 1.1696 & 1.1648 & 1.1688 & 1.1711 \\
\hline & 1.5520 & & & 1.5614 \\
\hline & 1.9504 & & 1.9503 & 1.9518 \\
\hline \multirow[t]{10}{*}{2.3288} & $2.3384,2.3681$ & 2.3288 & 2.3369 & 2.3421 \\
\hline & & & & 2.7325 \\
\hline & & & & 3.1228 \\
\hline & $3.5068,3.5392$ & & 3.5072 & 3.5132 \\
\hline & & & & 3.9035 \\
\hline & & & & 4.2939 \\
\hline & 4.6755 & & & 4.6842 \\
\hline & & & & 5.0746 \\
\hline & & & $5.4567,5.5913$ & 5.4649 \\
\hline & 5.8479 & & 5.8080 & 5.8553 \\
\hline
\end{tabular}


TABLE II. Counterpart of Table I with the rough tolerance but using values of $d$ out of its optimal interval.

\begin{tabular}{|c|c|c|c|}
\hline \multicolumn{3}{|c|}{ Rough tolerance (18) } & \multirow{2}{*}{$\begin{array}{c}\text { Attractor } \\
\ldots\end{array}$} \\
\hline DMD-50 & DMD-450 & DMD-475 & \\
\hline & 0.3869 & & 0.3904 \\
\hline & & & 0.7807 \\
\hline \multirow[t]{3}{*}{1.1654} & $1.1696,1.21,1.30,1.13$ & 1.1680 & 1.1711 \\
\hline & 1.5520 & & 1.5614 \\
\hline & 1.9504 & & 1.9518 \\
\hline \multirow[t]{6}{*}{2.3253} & $2.3384,2.3681$ & & 2.3421 \\
\hline & & & 2.7325 \\
\hline & & & 3.1228 \\
\hline & 3.5068, 3.5392 & & 3.5132 \\
\hline & & & 3.9035 \\
\hline & & & 4.2939 \\
\hline \multirow[t]{4}{*}{4.6740} & 4.6755 & & 4.6842 \\
\hline & & & 5.0746 \\
\hline & & & 5.4649 \\
\hline & 5.8479 & & 5.8553 \\
\hline
\end{tabular}

have been performed using DMD-50, DMD-450, and DMD475 with the rough tolerance. These values are out of the range of the optimal value for $d$, defined for this problem as $d \in[200,300]$. The optimal $d$ is set as the value in which the error of reconstruction of the original data is minimum. ${ }^{15}$ Table II shows the results obtained. As seen the performance of HODMD is worse when the value of $d$ is not optimal, but the method still captures frequencies similar to the ones previously obtained (attractor frequencies). In the three cases, HODMD calculates the fundamental frequency obtained in the experimental measurements. However, the 1/3-subharmonic frequency is only obtained in the case at DMD-450. The fundamental frequency and its first harmonic are calculated with larger accuracy in the case at DMD-50 than at DMD-1, in good agreement with the better performance of HODMD than the standard DMD, but the solution is still incomplete compared to the results obtained with larger $d$. At DMD-450, the method calculates more frequencies, but the fundamental

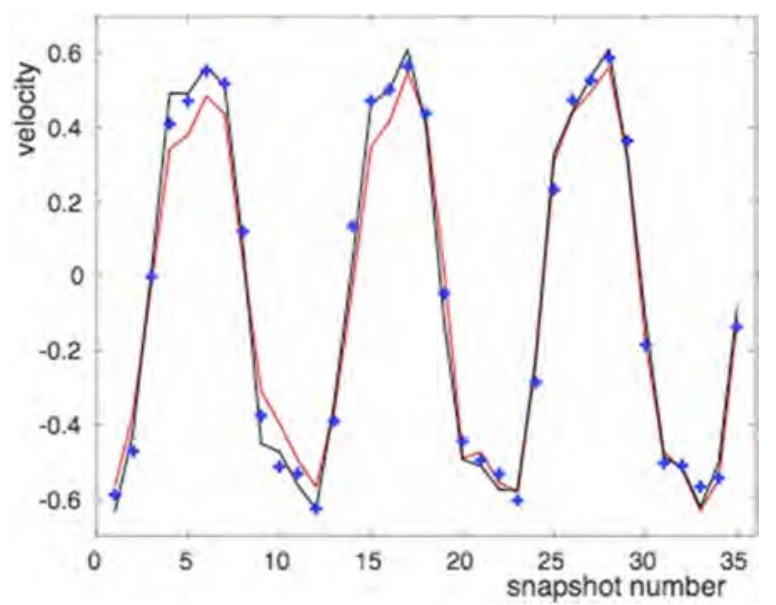

FIG. 5. Time evolution of the normal velocity in the cylinder wake in a single representative point. The figure compares the signal in the time interval (575, 591) (red line), and the signal (black line), and its extrapolation using DMD250 (blue crosses) in the attractor in the time interval (2900, 2916). frequency and its first and second harmonics are doubled. Finally, the most extreme case, DMD-475, only calculates the fundamental frequency, but with larger accuracy than DMD1. It is remarkable that the number of snapshots used for this calculation is 500 , so using DMD-475 means that only 25 snapshots are left free for the construction of the modified Koopman equation (13). The proved robustness of HODMD makes this tool as suitable to any type of calculations, even though when the calibration of $d$ carried out by the user is not optimal.

Figure 5 shows the time evolution of the velocity field in a single representative point of the domain, on a period of the 1/3-subharmonic frequency, and compares the region in which the analysis is performed (time $\sim 575$ ), the attractor (time $\sim 2900$ ), and the extrapolation to the attractor using DMD-250 and the more precise tolerance defined in Eq. (18).
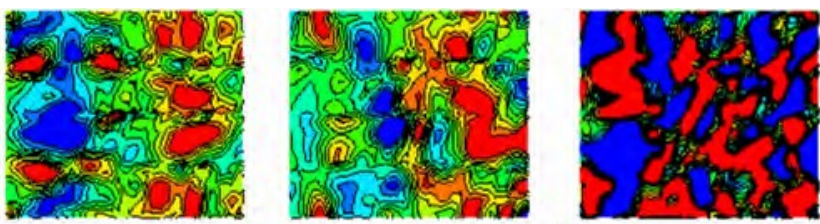

DMD-1 using the rough tolerance
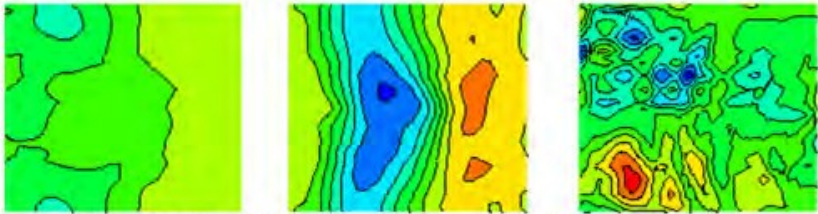

DMD-250 using the rough tolerance
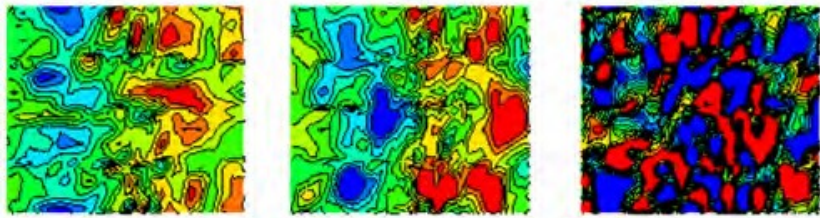

DMD-1 using the precise tolerance
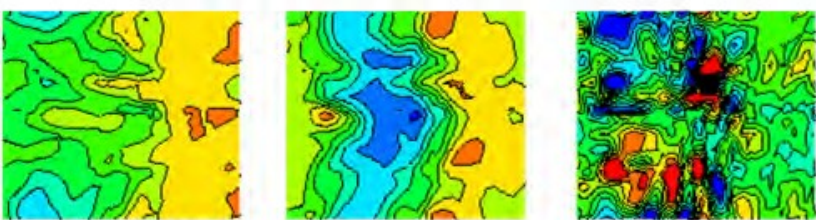

DMD-250 using the precise tolerance
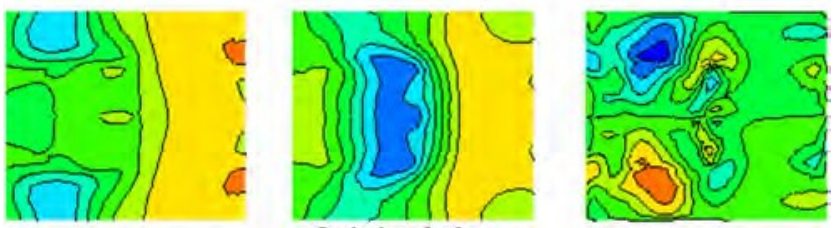

Original data

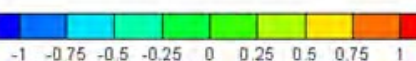

FIG. 6. Reconstruction (time 575). From top to bottom: a representative snapshot showing the velocity vector reconstructed using DMD-1, DMD-250 with the rough tolerances defined in Eq. (18), DMD-1, DMD-250 with the precise tolerances defined in Eq. (18), and the original data. From left to right: streamwise, normal, and spanwise velocity. Data normalized with the maximum velocity value of the real signal. 

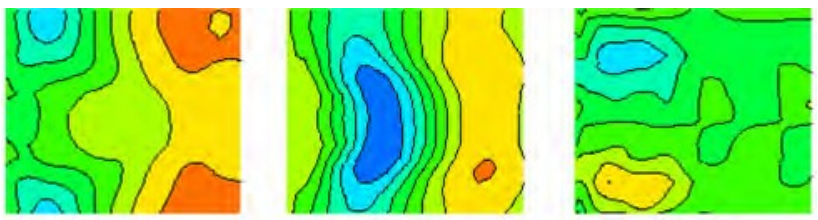

DMD-1 using the rough tolerance
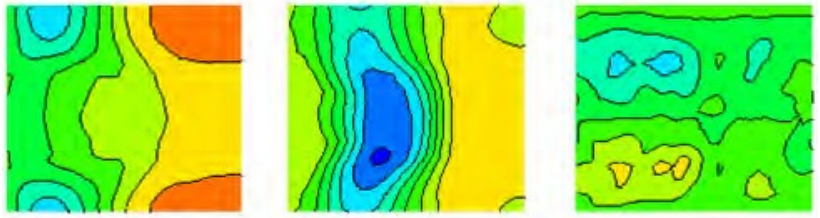

DMD-250 11sing the rough tolerance
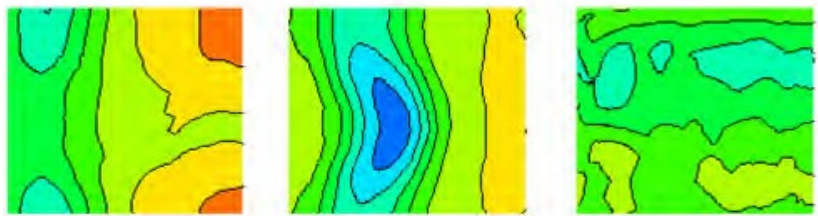

DMD-1 using the precise tolerance
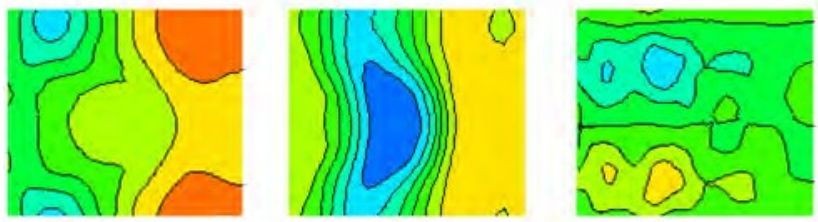

DMD-250 nsing the precise tolerance
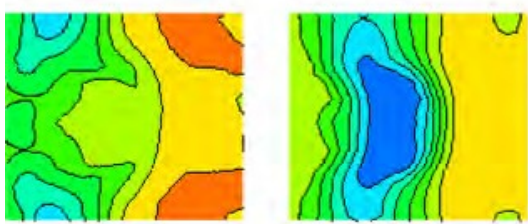

Original data
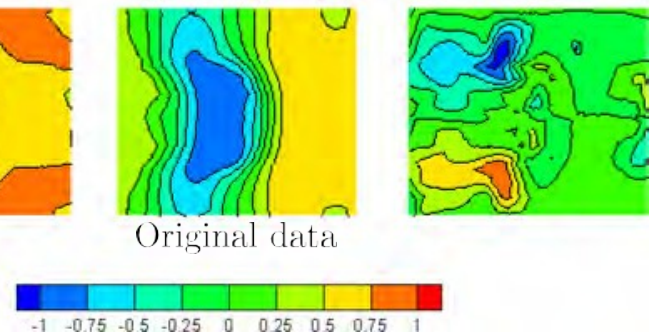

FIG. 7. Counterpart of Fig. 6 but for extrapolation to the attractor (time 2900).

As seen, the difference among the transient solution and the converged solution in the attractor is around $12 \%$, but the extrapolation solution matches quite well the attractor signal (maximum error smaller than 5\%). This fact makes worthy the use of this technique for extrapolation instead of continuing the integration of the Navier-Stokes equations, whose computational cost would be $2900 / 575-5$ times larger.

However, when the computational domain is larger, it is necessary to calculate global RMS errors, since the dynamics calculated in transient flows may not be highly accurate (some modes are missing or the frequencies calculated are not exact), and the results could affect some spatial areas more than others. Figures 6 and 7 show the outcome of the HODMD method (using data from the transient stage) for a representative snapshot taken at times close to 575 and 2900 of the original data (these times have been adapted to take the same phase of the signal in both cases), respectively. Let us note that both permanent and transient modes are used for reconstruction (high amplitude modes), but only permanent modes are used for extrapolation. Figure 6 gives the reconstruction of the data using DMD-1 and DMD-250, using both the rough and more precise tolerances defined in Eq. (18). The results in this figure indicate that the reconstruction of the original data (in the transient stage) fails using both DMD-1 and DMD-250, although the performance of DMD-250 is much better, since the method at least is capable of reproducing the largest scales and their intensities. In addition, the results obtained using the rough tolerance are better. The reason is that, when the analysis is performed in transient flow, a large quantity of modes are spurious and, the lower the tolerance, the larger quantity of spurious modes retained (all the modes shown in Figs. 2 and 3, crosses and circles, are used for this reconstruction). Figure 7 gives the extrapolation to the attractor using the same techniques. As can be seen, the outcomes of the method are much better now. On the one hand, the qualitative results obtained with DMD-1 and DMD-250 are both acceptable, though DMD-250 shows a slightly better performance in all these cases. On the other hand, the results obtained with the more precise tolerance are also slightly better in all these cases. So, it is possible to conclude that DMD-1 and DMD-250 (based on data from the transient stage) better reproduce the attractor than their own reconstruction. The reason is that the transient modes used for reconstruction (but eliminated for extrapolation) are high amplitude modes, but spurious. A similar behaviour is found in the case of complex experimental data in which, at most, only the two modes with higher amplitudes can be used for reconstruction, ${ }^{15}$ unless special treatment for noise is used. ${ }^{17}$ Thus, transient modes can be compared with noise and need to be treated in a similar way.

Finally, the temporal root mean square (RMS) errors, calculated in a period of the smaller frequency $(\omega=0.3904)$, give a quantitative estimation of the performance of the method. As in Fig. 5, the calculation of these errors uses 34 snapshots. Figures 8 and 9 show the RMS errors calculated in the complete domain showing the regions of maximum and minimum errors. As expected, this error is smaller in the
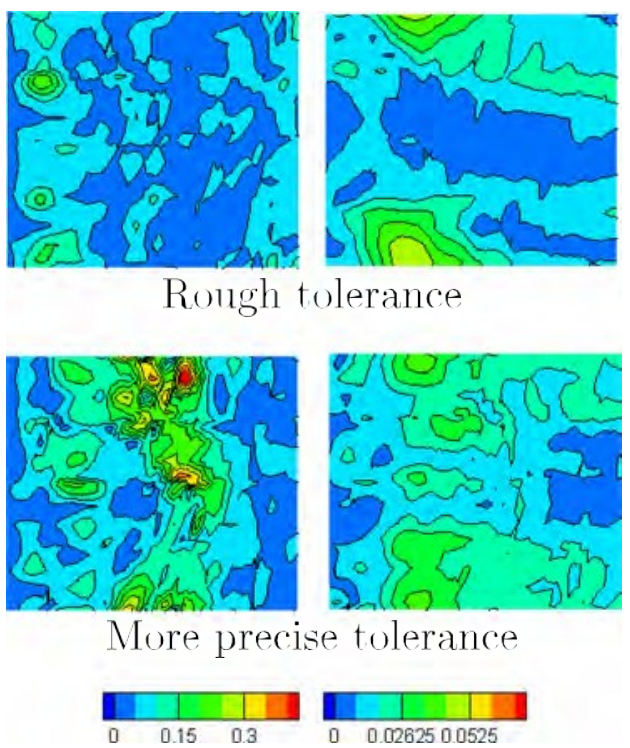

FIG. 8. Distribution of mean RMS errors in a period, calculated with the lower frequency, in the reconstruction of the original data using DMD-1 (left) and DMD-250 (right). 


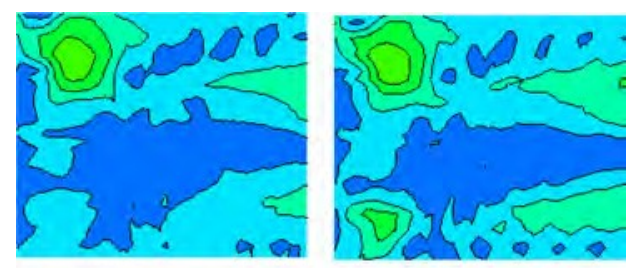

Rough tolerance

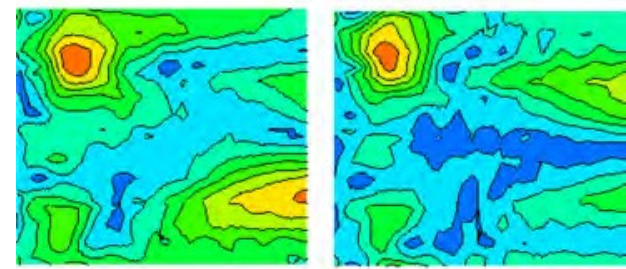

More precise tolerance

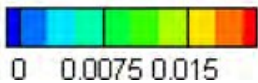

FIG. 9. Counterpart of Fig. 8 but for extrapolation results.

cases of extrapolation than reconstruction, which is consistent with the plots in Figs. 7 and 8. This maximum value is $\sim 1.5 \times 10^{2}$ for extrapolation using both DMD-1 and DMD250 and the rough tolerances, while it is $\sim 2 \times 10^{2}$ with the more precise tolerance, again using both DMD- 1 and DMD250. For reconstruction, using DMD-1, the maximum error is $\sim 35 \times 10^{1}$ and $\sim 4 \times 10^{1}$ for the rough and more precise tolerances, respectively, while using DMD-250 it is $\sim 6$ $\times 10^{2}$ for both tolerances. Thus, DMD-250 is more robust than DMD-1. Even though the velocity contours used in extrapolation are quite similar when DMD-1 and DMD-250 are used, the global errors are maintained slightly smaller using DMD250. However, DMD-1 also seems a suitable tool used for these purposes. Finally, let us mention that although the solution is not exact, the time reduction makes it worthy to use HODMD for extrapolation. If one wants to reduce this extrapolation error, we suggest using this method as a reduced order model with the following three steps: (i) run the code for a short period of time, (ii) extrapolate to the attractor (very large time), and (iii) fix the aforementioned errors running the code for a very short period of time, starting from the predicted attractor solution. This idea is beyond the scope of the present article but it remains as an open topic to be developed in future studies.

Obviously, the outcomes of the method improve as the time at which the snapshots are collected is more advanced and/or the number of snapshots used is larger. This issue is considered in Figs. 10 and 11. In particular, Fig. 10 shows the frequencies, amplitudes, and growth rates calculated by DMD1 and DMD700 in a more advanced time region. The new value $d=700$ to apply the DMD- $d$ algorithm has been selected after some calibration. As in the remaining applications, HODMD above, this value of $d$ can be increased or decreased by 50 without significant changes in the performance of the method.

On the other hand, Fig. 11 shows analogous DMD computations performed in the far field plane described in Eq. (17). As in the previously presented application, circles and crosses correspond to the permanent and transient modes, respectively. As expected, the gap dividing transient and permanent modes is now slightly larger (as time passes by, the transient dynamics decays and the permanent dynamics is more converged, increasing the distance between both solutions). In addition, the number of transient modes calculated in the far field is
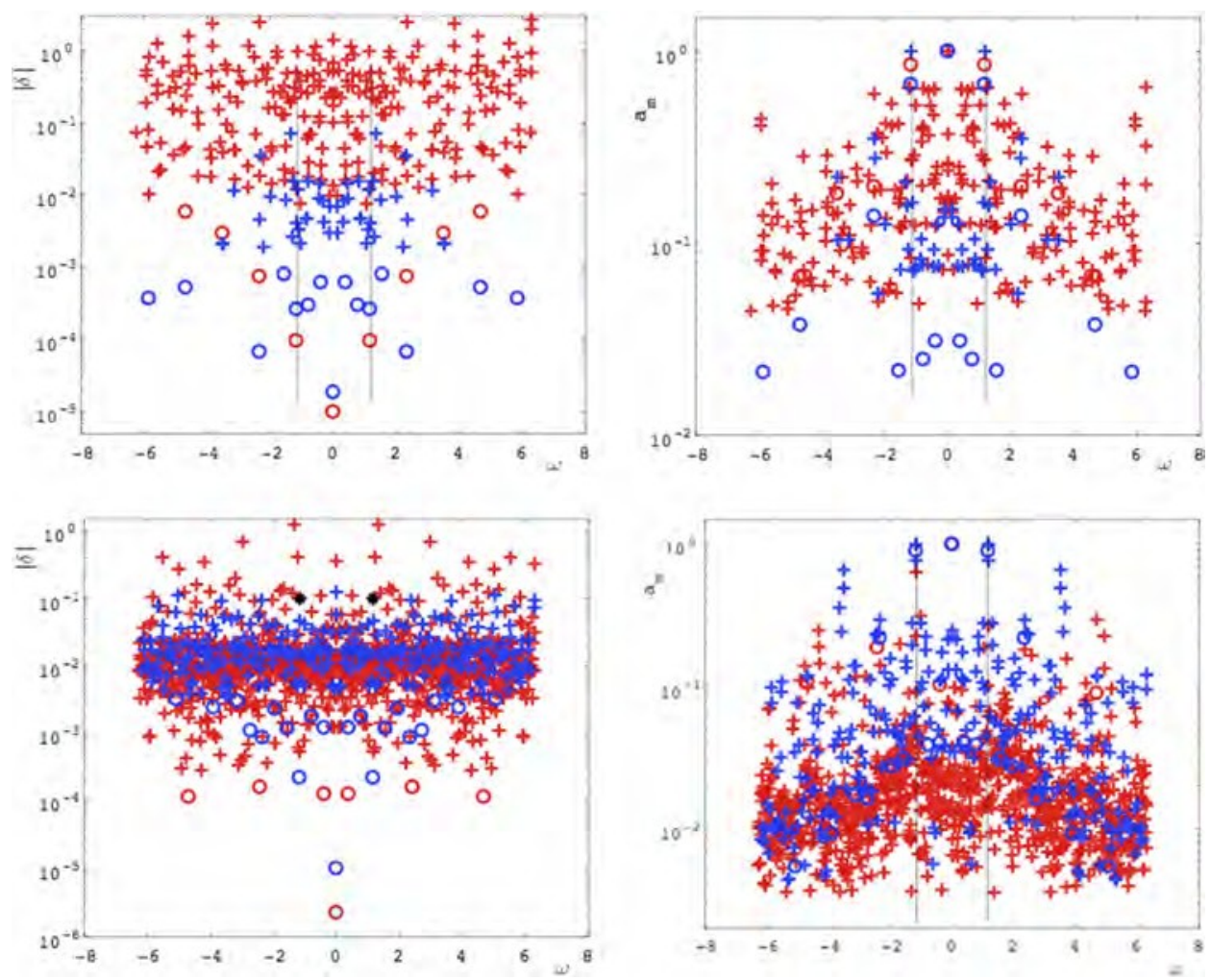

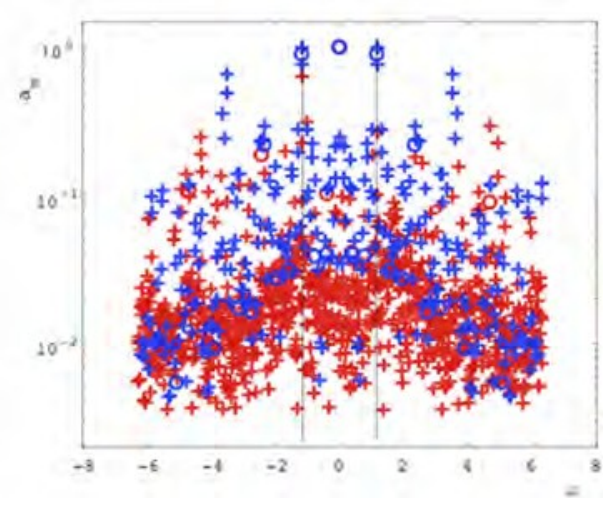

FIG. 10. Counterpart of Fig. 2, but using DMD-700 and DMD-1 in a set of 1000 snapshots starting at time 825 and considering the rough tolerances (top) and the more precise tolerances (bottom) defined in Eq. (18). 

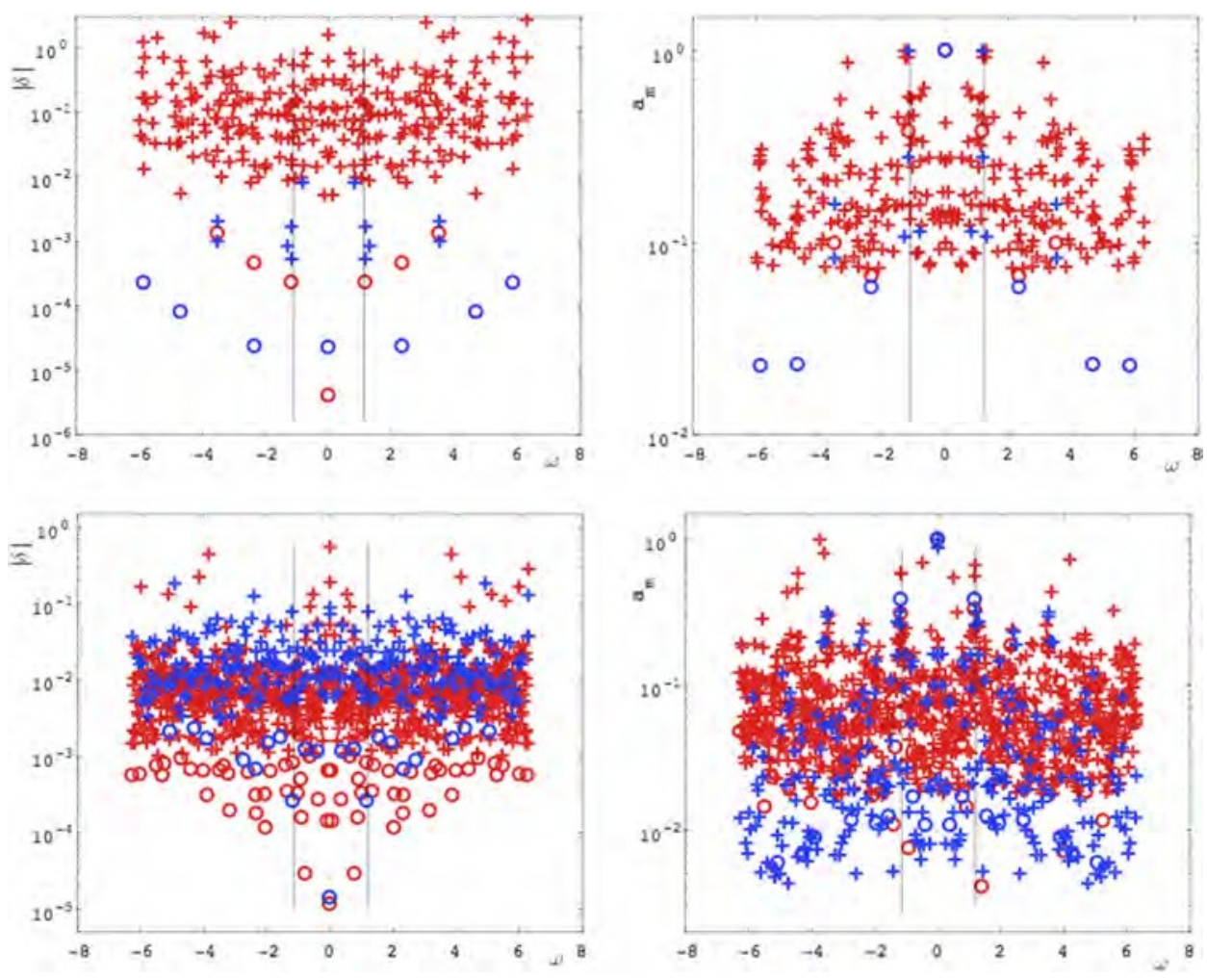

FIG. 11. Counterpart of Fig. 10, but considering the $\mathrm{y}=0$-far-field defined in Eq. (17).

also larger, since the number of snapshots now collected has increased. Table III gives the calculated permanent mode frequencies, shown in Figs. 10 and 11, and the results obtained in the attractor. These results show the robustness and good performance of DMD-700 when the conditions change. As expected, the performance of DMD-700 improves with the more precise tolerance as far as the data collected are closer to the attractor, since the method is capable of identifying and removing spatial redundancies and make profit of the real spatial modes. For this reason, DMD-700 identifies a larger number of the attractor patterns in both the near field and the far field, specially in the far field, since the computational domain is larger (the more spatial information, the better performance of HODMD). On the contrary, the performance of DMD-1 is neither as robust or accurate in any of these cases since (i) using the rough tolerance, DMD-1 only identifies the experimental frequency and their harmonics (only the high amplitude frequencies) and (ii) using the more precise

TABLE III. Summary of the permanent frequencies $\omega$ identified in Figs. 2 and 3 and the attractor frequencies. Reference frequency (experiment): $\omega_{\exp }=1.1624$.

\begin{tabular}{|c|c|c|c|c|c|c|c|c|}
\hline \multicolumn{4}{|c|}{ Near field } & \multicolumn{4}{|c|}{ Far field } & \multirow{3}{*}{$\begin{array}{c}\text { Attractor } \\
\text { Attractor } \\
\ldots\end{array}$} \\
\hline \multicolumn{2}{|c|}{ Rough tolerance } & \multicolumn{2}{|c|}{ More precise } & \multicolumn{2}{|c|}{ Rough tolerance } & \multicolumn{2}{|c|}{ More precise } & \\
\hline DMD-1 & DMD-d & DMD-1 & DMD-d & DMD-1 & DMD-d & DMD-1 & DMD-d & \\
\hline & 0.3886 & 0.3853 & 0.3869 & & & 0.3603 & 0.3883 & 0.3904 \\
\hline & 0.7800 & & 0.7816 & & & 0.7834 & 0.7808 & 0.7807 \\
\hline \multirow[t]{3}{*}{1.1700} & 1.1689 & & 1.1695 & 1.1699 & & 0.0726 & 1.1698 & 1.1711 \\
\hline & 1.5547 & & 1.5562 & & & 1.3457 & 1.5561 & 1.5614 \\
\hline & & & 1.9508 & & & 1.0158 & 1.9493 & 1.9518 \\
\hline \multirow[t]{3}{*}{2.3401} & 2.3373 & 2.4180 & 2.3392 & 2.3400 & 2.3407 & 2.3250 & 2.3398 & 2.3421 \\
\hline & & & 2.7245 & & & 2.3372 & 2.7250 & 2.7325 \\
\hline & & & 3.1193 & & & 3.1658 & 3.1180 & 3.1228 \\
\hline \multirow[t]{3}{*}{3.5109} & & & & 3.5098 & & & 3.5094 & 3.5132 \\
\hline & & & 3.8934 & & & 3.8835 & 3.8936 & 3.9035 \\
\hline & & & & & & & 4.2861 & 4.2939 \\
\hline \multirow[t]{4}{*}{4.6802} & 4.6811 & 4.6812 & & & 4.6808 & & 4.6790 & 4.6842 \\
\hline & & & 5.0623 & & & & 5.0617 & 5.0746 \\
\hline & & & & & & & & 5.4649 \\
\hline & 5.8517 & & & & 5.8513 & & 5.7753 & 5.8553 \\
\hline
\end{tabular}


TABLE IV. Counterpart of Table III with the rough tolerance in the near field but using values of $d$ out of its optimal interval.

\begin{tabular}{|c|c|c|c|}
\hline \multicolumn{3}{|c|}{ Near field, rough tolerance (18) } & \multirow{2}{*}{$\begin{array}{c}\text { Attractor } \\
\ldots\end{array}$} \\
\hline DMD-500 & DMD-900 & DMD-975 & \\
\hline & & & $\begin{array}{l}0.3904 \\
0.7807\end{array}$ \\
\hline \multirow[t]{3}{*}{1.1715} & 1.1701 & 1.1703 & 1.1711 \\
\hline & & & 1.5614 \\
\hline & & & 1.9518 \\
\hline \multirow[t]{3}{*}{2.3538} & 2.3406 & 2.3410 & 2.3421 \\
\hline & & & 2.7325 \\
\hline & & & 3.1228 \\
\hline \multirow[t]{7}{*}{3.5117} & 3.5107 & 3.5139 & 3.5132 \\
\hline & & & 3.9035 \\
\hline & & & 4.2939 \\
\hline & 4.6803 & & 4.6842 \\
\hline & & & 5.0746 \\
\hline & & & 5.4649 \\
\hline & & & 5.8553 \\
\hline
\end{tabular}

tolerance, DMD-1 is not even capable of capturing the experimental frequency, but divides this mode into three distinct modes. This fact makes DMD- $d$ a suitable tool to be used for pattern identifications when the dynamics are not converged (numerical simulations or complex experiments), when the data collected are limited in space (experiments) or when the data are noisy, since noise can be somehow equated to transient dynamics (experiments).

Again, in order to show the robustness of HODMD, the same calculations have been performed using DMD-500, DMD-900, and DMD-975 with the rough tolerance in the near field. These values are out of the range of the optimal value for $d$, defined for this problem as $d \in[650,750]$. As in the previous case, the optimal $d$ is set as the value in which the error of reconstruction of the original data is minimum. Table IV shows the results obtained. As seen, in the three cases, HODMD captures the fundamental frequency obtained in the

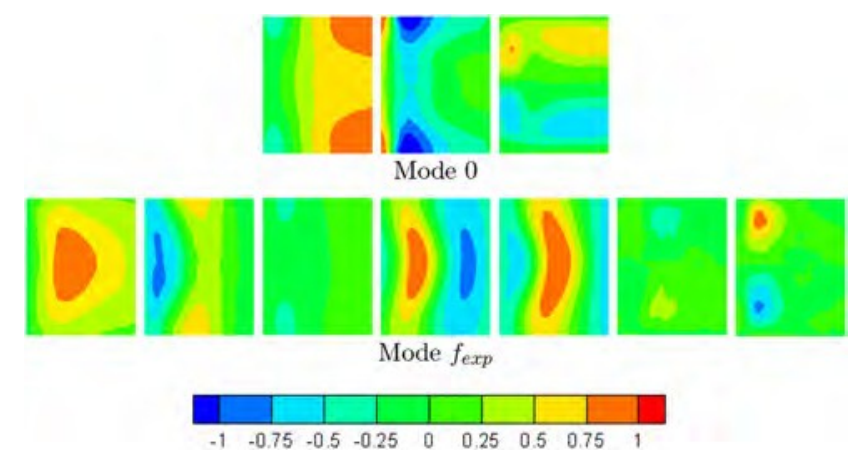

FIG. 12. The mode associated with the mean flow [top, plotting, from left to right, $\left(u_{x}\right),\left(u_{y}\right)$, and $\left.\diamond\left(u_{z}\right)\right]$ and the mode associated with the experimental frequency [bottom, plotting, from left to right, $\left(u_{x}\right), 5\left(u_{x}\right), \diamond\left(u_{y}\right)$, $\left.5\left(u_{y}\right),\left(u_{z}\right), 5\left(u_{z}\right)\right]$.

experiments, and its first and second harmonics. The third harmonic is also obtained at DMD-900. The frequencies are calculated with high accuracy in all these cases, but in any of them is neither possible to identify the frequency related to the 1/3-subharmonic nor its harmonics. Once more, the robustness of HODMD methodology has been put in evidence; nevertheless, the calibration process carried out to obtain the optimal value of $d$ is necessary.

Let us now consider some of the DMD modes, as calculated using DMD-700 with the more precise tolerances. The dominant modes are the mean flow (only real part) and the experimental frequency, which are considered in Fig. 12. As can be seen, the streamwise component of the mean flow preserves very well the up-down reflection symmetry, but the normal and spanwise components preserve this symmetry only approximately. This is due to the combination of numerical errors and DMD errors, which manifest themselves in the cross-flow velocity components, which for the mean flow are much smaller than the streamwise component. Concerning the mode associated with the experimental frequency, the lower plots in Fig. 12 give not only the real and imaginary parts of the three velocity components but also the intensity of the
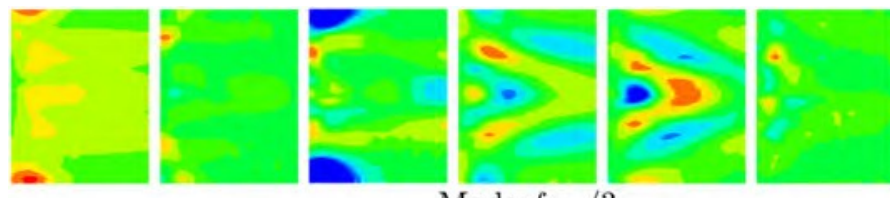

Mode $f_{\exp } / 3$
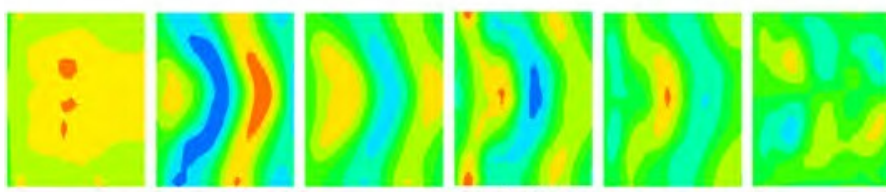

Mode $2 f_{\exp }$
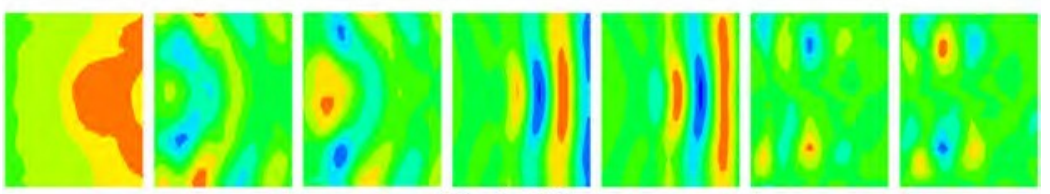

Mode $3 f_{\text {exp }}$
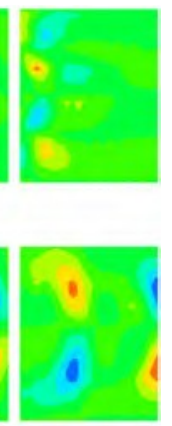

FIG. 13. Counterpart of Fig. 12, considering the indicated modes.

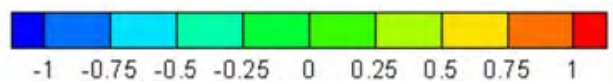


mode, defined as

$$
I=\mid \overline{\left.u_{x}\right|^{2}+\left|u_{y}\right|^{2}+\left|u_{z}\right|^{2}}
$$

As can be seen, the up-down symmetry is preserved in those regions where the intensity is larger, as expected. Obviously, the real and imaginary parts are not proportional to each other, which is consistent with the traveling character of the pattern, which is slightly curved in the spanwise direction.

Some of the remaining modes are considered in Fig. 13, where it can be seen that (i) again the real and imaginary parts are far from being proportional to each other and (ii) the up-down symmetry is generally not well-preserved, which invoking Fig. 4 is a consequence of the fact that these modes exhibit much lower amplitudes than those in Fig. 12. On the other hand, it is interesting to note that the new $f_{\text {exp }} / 3$ mode exhibits a chevron-like pattern, suggesting a relation between these results and the experimental results obtained by Williamson. ${ }^{27}$ As predicted by Williamson, who performed experiments in a water tunnel to study a cylinder which is infinitely long, the chevron-like patterns were found when the flow over the span was matching the end conditions of such a cylinder. In the numerical simulations, a periodicity condition is imposed on the two sides of the computational domain. Thus, the three-dimensional flow is enforced to appear with a specific wave length. This suggests that the mode represented by the chevron-like pattern, which is a low amplitude mode, is necessary to adjust the solution to the specific length $L_{z}$.

\section{CONCLUDING REMARKS}

The extrapolation from the transient behavior approaching an attractor to the attractor itself has been considered using DMD-like methods, namely, standard DMD, and its recently introduced higher order extension, ${ }^{16}$ HODMD. This new method has been briefly described and its performance is compared with classical DMD in the analysis of numerical simulations on the three-dimensional cylinder wake.

The conclusion is that the intended extrapolation is feasible using DMD-like methods, especially using HODMD. In particular, the relevant frequencies involved in the attractor are fairly well anticipated, as are the involved patterns. This conclusion makes these methods potentially useful to both save computational time in numerical simulations and reduce the storage needs in PIV measurements, especially near bifurcation points, where the approach to the attractor can be extremely slow.

\section{ACKNOWLEDGMENTS}

This research was partially supported by the Spanish Ministry of Economy and Competitiveness, under Grant No. TRA2016-75075-R. The authors are indebted to the anonymous referees for some useful comments that helped to improve the presentation of the article.
${ }^{1}$ Bagheri, S., “Koopman-mode decomposition of the cylinder wake,” J. Fluid Mech. 726, 596-623 (2013).

${ }^{2}$ Barkley, D. and Henderson, R., "Three-dimensional Floquet şț̨̨ility analysis of the wake of a circular cylinder,” J. Fluid Mech. , 215-241 (1996).

${ }^{3}$ Blackburn, H. M., Marques, F., and Lopez, J. M., "Symmetry breaking of two-dimensional time-periodic wakes,” J. Fluid Mech. 522, 395-411 (2005).

${ }^{4}$ De Lathawer, L., De Moor, B., and Vandewalle, J., “A multilinear singular value decomposition,” SIAM J. Matrix Anal. Appl. 21, 1253-1278 (2000). ${ }^{5}$ Ferrer, E., de Vicente, J., and Valero, E., "Low cost 3D global instability analysis and flow sensitivity based on dynamic mode decomposition and high-order numerical tools,” Int. J. Numer. Methods Fluids 76(3), 169-184 (2014).

${ }^{6}$ See http://gmsh.info/ for more information to download Gmsh.

${ }^{7}$ Gómez, F., Le Clainche, S., Paredes, P., Hermanns, M., and Theofilis, V., "Four decades of studying global linear instability: Progress and challenges,” AIAA J. 50(12), 2731-2743 (2012).

${ }^{8}$ Hammache, M. and Gharib, M., "An experimental study of the parallel and oblique vortex shedding from circular cylinders," J. Fluid Mech. 232, 567-590 (1991).

${ }^{9}$ Jackson, C. P., "A finite-element study of the onset of vortex shedding in ow past variously shaped bodies,” J. Fluid Mech. 182, 23-45 (1987).

${ }^{10}$ Kolda, T. G. and Bader, B. W., "Tensor decompositions and applications," SIAM Rev. 51, 455-500 (2000).

${ }^{11}$ Koopman, B., "Hamiltonian systems and transformations in Hilbert space," Proc. Natl. Acad. Sci. U. S. A. 17, 315-318 (1931).

${ }^{12}$ Le Clainche, S., Li, J. I., Theofilis, V., and Soria, J., "Flow around a hemisphere-cylinder at high angle of attack and low Reynolds number. Part I: Experimental and numerical investigation,” Aerosp. Sci. Technol. 44, 77-87 (2015).

${ }^{13}$ Le Clainche, S., Rodríguez, D., Theofilis, V., and Soria, J., "Flow around a hemisphere-cylinder at high angle of attack and low Reynolds number. Part II: POD and DMD applied to reduced domains,” Aerosp. Sci. Technol. 44, 88-100 (2015).

${ }^{14}$ Le Clainche, S., Rodríguez, D., Theofilis, V., and Soria, J., "Formation of three-dimensional structures in the hemisphere-cylinder," AIAA J. 54(12), 3884-3894 (2016).

${ }^{15}$ Le Clainche, S., Sastre, F., Vega, J. M., and Velazquez, A., "Higher order dynamic mode decomposition applied to study flow structures in noisy PIV experimental data," AIAA Paper 2017-3304, 2017.

${ }^{16}$ Le Clainche, S. and Vega, J. M., "Higher order dynamic mode decomposition,” SIAM J. Appl. Dyn. Syst. 16(2), 882-925 (2017).

${ }^{17}$ Le Clainche, S., Vega, J. M., and Soria, J., "Higher order dynamic mode decomposition of noisy experimental data: The flow structure of a zero-netmass-flux jet," Exp. Therm. Fluid Sci. 88, 336-353 (2017).

${ }^{18}$ Moreno, R., Le Clainche, S., Vega, J. M., and Taylor, P., "Flight flutter testing using higher order dynamic mode decomposition," presented at the International Forum of Aeroelascity and Structural Dynamics (IFASD 2017), Como, Italy, 25-28 June 2017.

${ }^{19}$ See https://nek5000.mcs.anl.gov/ for more information to download Nek5000.

${ }^{20}$ Pérez, J. M., Le Clainche, S., and Vega, J. M., “Alternative three-dimensional instability analysis of the wake of a circular cylinder," AIAA Paper 20174021, 2017.

${ }^{21}$ Provanasal, M., Mathis, C., and Boyer, L., "Bénard-von Kármán instability: Transient and forcing regimes,” J. Fluid Mech. 182, 1-22 (1987).

${ }^{22}$ Rowley, C. W., Mezić, I., Bagheri, S., Schlatter, P., and Henningson, D. S., "Spectral analysis of nonlinear flows," J. Fluid Mech. 641, 115-127 (2009).

${ }^{23}$ Schmid, P. J., "Dynamic mode decomposition of numerical and experimental data,” J. Fluid Mech. 656, 5-28 (2010).

${ }^{24}$ Schmid, P. J., "Applications of the dynamic mode decomposition to experimental data,” Exp. Fluids 50, 1123-1130 (2011).

${ }^{25}$ Takens, F., in Detecting Strange Attractors in Turbulence, Lecture Notes in Mathematics, edited by Rand, D. A. and Young, L.-S. (Springer-Verlag, 1981), pp. 366-381.

${ }^{26}$ Tucker, L. R., "Some mathematical notes on three-mode factor analysis," Psychometrika 31, 279-311 (1996).

${ }^{27}$ Williamson, C. H. K., "Oblique and parallel modes of vortex shedding in the wake of a circular cylinder at low Reynolds number,” J. Fluid Mech. 206, 579-627 (1989).

${ }^{28}$ Williamson, C. H. K., "Vortex dynamics in the cylinder wake,” Annu. Rev. Fluid. Mech. 28, 477-539 (1996). 